\title{
A Stimulation Method to Assess the Contractile Status of the Lumbar Extensors in a Seated Posture
}

\author{
Bochen Jia, ${ }^{1}$ Maury A. Nussbaum, ${ }^{2}$ and Michael J. Agnew ${ }^{2}$ \\ 1 Industrial and Manufacturing Systems Engineering, University of Michigan - Dearborn, Dearborn, Michigan, USA \\ 2 Department of Industrial and Systems Engineering, Virginia Tech, Blacksburg, Virginia, USA
}

\begin{abstract}
The purpose of present study was to develop and evaluate methods to assess stimulation responses of the lumbar extensors, as part of a longer-term goal of detecting fatigue during prolonged sitting. Three stimulation frequencies $(2,5$, and $8 \mathrm{~Hz})$ were tested in separate stages, which include 3 stimulation trains and 4 sampling blocks. Repeated measures analyses of variance were used to determine whether any significant differences in mean stimulation responses occurred with respect to stimulation frequency, sampling block, and stimulation train. Reliability of measured stimulation responses was assessed within and between sampling blocks using intraclass correlation coefficients. Stimulation frequencies significantly affected the stimulation responses and time-to-potentiation differed between the 3 stimulation frequencies; it was highest for $2 \mathrm{~Hz}$ stimulation. All 3 stimulation frequencies resulted in excellent reliability within and between sampling blocks. Use of the current protocol at $2 \mathrm{~Hz}$ is recommended as appropriate to measure the lumbar extensors status during prolonged sitting. ( $) 2014$ Wiley Periodicals, Inc.
\end{abstract}

Keywords: Muscle stimulation; Stimulation frequency; Lumbar extensors

\section{INTRODUCTION}

Modern living increases the tendency to have a more sedentary lifestyle that involves prolonged sitting (Ford, Kohl, Mokdad, \& Ajani, 2005; Varo et al., 2003). In the United States, for example, an earlier report indicated that more than $60 \%$ of adults are rarely physically active and $20 \%$ of all adults are completely sedentary (U.S. Department of Health and $\mathrm{Hu}-$ man Services, 1996). Current estimates are consistent in terms of leisure-time physical activity in the United States (Pleis, Lucas, \& Ward, 2009) and more gener-

Correspondence to: Maury A. Nussbaum, Department of Industrial and Systems Engineering, Virginia Tech, 250 Durham Hall (0118), Blacksburg, VA 24061. Phone: 540-231-6053; e-mail: nussbaum@vt.edu

Received: 21 June 2012; revised 28 February 2014; accepted 7 March 2014

View this article online at wileyonlinelibrary.com/journal/hfm DOI: $10.1002 / \mathrm{hfm} .20584$ ally that less than one third of adults worldwide are physically active (Hallal et al., 2012). Existing evidence indicates that prolonged sitting postures compromises blood flow, increases intradiscal loads, and reduces muscle oxygenation (Vollestad, 1997). Such physiological changes can cause lumbar extensor fatigue that in turn may contribute to low back pain (Dankaerts, O’Sullivan, Burnett, \& Straker, 2006; Kolich, Mohamed, \& Taboun, 2001; NIOSH, 1997; O’Sullivan, Mitchell, Bulich, Waller, \& Holte, 2006; Womersley \& May, 2006). Postural muscles are continuously activated to stabilize the sitting posture, though relatively low levels of muscle activity ( $<\sim 10 \%$ of maximal muscle capacity) are involved (Mork \& Westgaard, 2005, 2009; van Dieën, de Looze, \& Hermans, 2001). Further, both objective and subjective signs of fatigue have been observed following $20 \mathrm{~min}$ or more of seated exposures (Blangsted, Sjøaard, Madeleine, Olsen, \& Søaard, 2005; Hakala, Rimpela, Saarni, \& Salminen, 2006; McLean \& Goudy, 2004; Sjøgaard, Kiens, Jørgensen, \& Saltin, 1986; Søgaard, Blangsted, Jørgensen, Madeleine, 
\& Sjøgaard, 2003; van Dieën, Westebring-van der Putten, Kingma, \& de Looze, 2009).

Objective signs of muscle fatigue during sitting have typically been obtained from electromyographic (EMG) measures, though the use of EMG in this context has associated challenges and has provided some conflicting results. As noted, relatively low contraction levels are involved, and these can be lower than that needed for EMG to detect fatigue-related changes (Gamet, Duchene, Garapon-Bar, \& Goubel, 1993; Nagata, Arsenault, Gagnon, Smyth, \& Mathieu, 1990; Oberg, Sandsjo, \& Kadefors, 1994; Oberg, Sandsjo, Kadefors, \& Larsson, 1992; Sood, Nussbaum, \& Hager, 2007). McLean and Goudy (2004) also indicated that during low contractions evidence of fatigue is inconsistent between different muscle groups and across individuals. While Søgaard et al. (2003) and van Dieën et al. (2009) demonstrated the feasibility of using EMG to detect muscle fatigue during low-level contractions (as low as $2 \% \mathrm{MVC}$ ), their results showed large intersubject variability.

A common alternative means to identify muscle fatigue is through external stimulation to obtain stimulation responses. In this approach, fatigue is indicated when stimulation responses fall significantly below initial, prefatigue levels (Binder-Macleod \& SnyderMackler, 1993; Edwards, Hill, Jones, \& Merton, 1977; Johnson, 1998; Johnson, Lehman, \& Rempel, 1995a). Muscle fatigue has been measured by comparing the decrement of response ratio between results to highfrequency $(50-100 \mathrm{~Hz})$ stimulation and low-frequency (1-20 Hz) stimulation (Byström \& Kilbom, 1991; Davies \& White, 1982; Edwards et al., 1977). Others have measured muscle fatigue using a single stimulation frequency (Cooper, Edwards, Gibson, \& Stokes, 1988; Johnson et al., 1995a). While a variety of stimulation protocols have been used, a greater loss in stimulation response can occur when evoked by low-frequency stimulation (Cooper et al., 1988; Fitch \& McComas, 1985). Low-frequency stimulation can also be advantageous since it is less likely to cause muscle fatigue or discomfort (Johnson et al., 1995a). Low-frequency muscle stimulation has been shown to be an effective method to assess muscle fatigue (Johnson et al., 1995a) and has been successfully applied to the forearm muscles (Johnson, Lehman, \& Rempel, 1995b, 1996; Mork \& Westgaard, 2005). Yet it remains unclear whether this approach would be similarly successful for assessing fatigue in the trunk musculature.
The current work is part of broader effort to use muscle stimulation (low frequency, specifically) to detect lumbar extensors fatigue during prolonged sitting. In this context, the present study was conducted to develop a reliable muscle stimulation protocol to measure stimulation responses in the lumbar extensors, specifically those muscles potentially fatigued during prolonged seated tasks. Several stimulation parameters and conditions can substantially affect stimulation response, thereby influencing the reliability of stimulation response measurements. During prolonged stimulation, stimulation responses undergo a gradual potentiation (Desmedt \& Hainaut, 1968), which, if not accounted for, can mask or confound other effects. For example, an increase in stimulation response amplitude due to potentiation and a decrease due to fatigue can occur simultaneously (Rassier \& MacIntosh, 2000) and has been observed during low-frequency fatigue caused by sustained low-level loading (Fowles \& Green, 2003). Potentiated stimulation responses may also be a more sensitive index of contractile fatigue than unpotentiated stimulation responses (Kufel, Pineda, \& Mador, 2002). The relationship between stimulation response generation and excitation also depends on stimulation frequency, which can also influence the pattern of potentiation (Rassier \& MacIntosh, 2000). In the current study, we evaluated the effects of several stimulation frequencies in terms of potentiation and the reliability of evoked stimulation responses. Results from this exploratory work were intended to facilitate the selection of stimulation protocols in future studies.

\section{METHODS}

\subsection{Overview}

A stimulation protocol to assess lumbar extensor stimulation responses was developed. Effective stimulation sites and stimulus intensity were identified for each participant since these may vary between individuals because of anatomical differences. Successive muscle stimulation can lead to a gradual increase in measured muscle responses, a phenomenon known as muscle potentiation (Desmedt \& Hainaut, 1968; Small \& Stokes, 1992). Since potentiation could lead to decreased reliability in stimulation responses, and/or mask fatiguerelated effects, it was considered important to control for these. To address this, a muscle conditioning train was completed to potentiate target muscles to a stable 


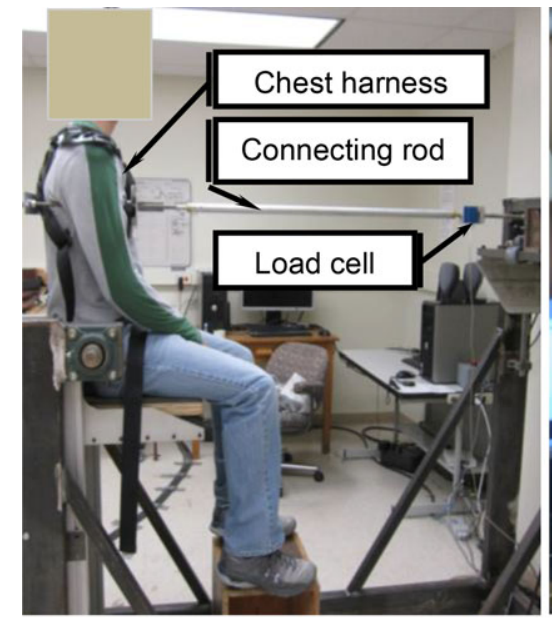

(A)

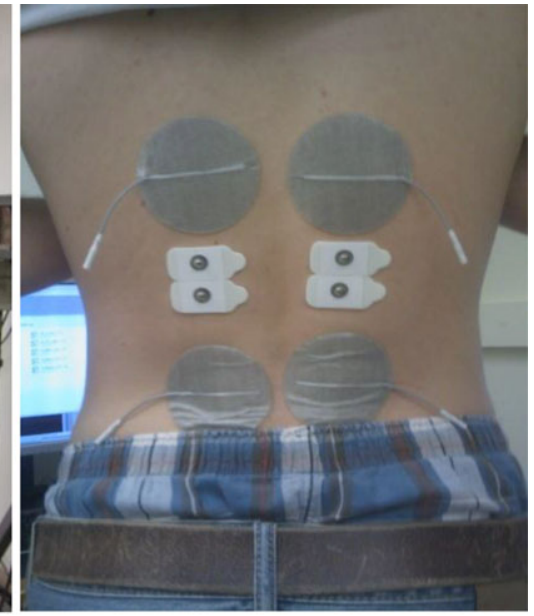

(B)

Figure 1 Illustration of $(A)$ experimental fixture setup demonstrating a participant in an upright sitting posture, and (B) the locations of dual-channel muscle stimulation electrodes (grey) and bipolar EMG electrodes (white).

status and was done using each of three stimulation frequencies. Stimulation responses of the lumbar extensors, represented by the forces measured from resulting (evoked) trunk extension, were measured in response to each stimulation frequency using the same stimulation protocol and via a load cell and fixture. Responses to the three stimulation frequencies were compared to help identify an appropriate stimulation protocol.

\subsection{Participants}

Six participants (gender balanced) were recruited and completed an informed consent process approved by the Virginia Tech Institutional Review Board. Their mean (standard deviation $[S D]$ ) age, height, body mass, and body mass index (BMI) were 28.2 (4.8) years, $170.3(8.7) \mathrm{cm}, 67.6(13.2) \mathrm{kg}$, and 23.2 (3.2), respectively. Individuals with body mass index $>30$ were excluded due to the potential difficulty in evoking reliable muscle contractions. All participants were physically active and had no self-reported musculoskeletal or neurological diseases within the past year that restricted their daily activities.

\subsection{Instrumentation}

The lumbar extensors were stimulated bilaterally using a dual-channel, current-controlled muscle stimulator (Grass S88, AstroMed, West Warwick, RI) in series with a stimulus isolation unit (SIU5, AstroMed, West Warwick, RI) and a constant current unit (CCU1,
AstroMed, West Warwick, RI). Two pairs of 7-cmdiameter bipolar stimulating electrodes (PALS Platinum Model 879300, Axelgaard Manufacturing Co., Fallbrook, CA) were placed bilaterally over the lumbar extensors (see below regarding stimulation site). During the experiment, participants sat in an experimental fixture (Figure 1A), in which motion of the pelvis was restrained using straps. An adjustable footrest was used to position the lower limb with right angles at the knee and ankle. Participants were positioned in a comfortable and relaxed upright sitting posture using a rigid rod connected to a chest harness at the T8 level and which contained an in-line load cell (Interface SM2000, capacity $=2,000 \mathrm{~N}$, Scottsdale, AZ). Stimulation responses were sampled at $1000 \mathrm{~Hz}$ from the load cell. To minimize voluntary muscle activity during stimulation response measurements, the erector spinae were monitored using two pairs of surface EMG electrodes that were placed bilaterally at the L3 level (Figure 1B). EMG signals were monitored visually throughout the experiments in real time. Muscle stimulation was started only after the observed level of muscle activation was (qualitatively) stabilized, specifically as reflected in no substantial increase/decrease in EMG levels.

\subsection{Experimental Procedures}

Participants' skin in the dorsal lumbar region was prepared following procedures as described by Cram and Rommen (1989). As needed, the skin surface where the 


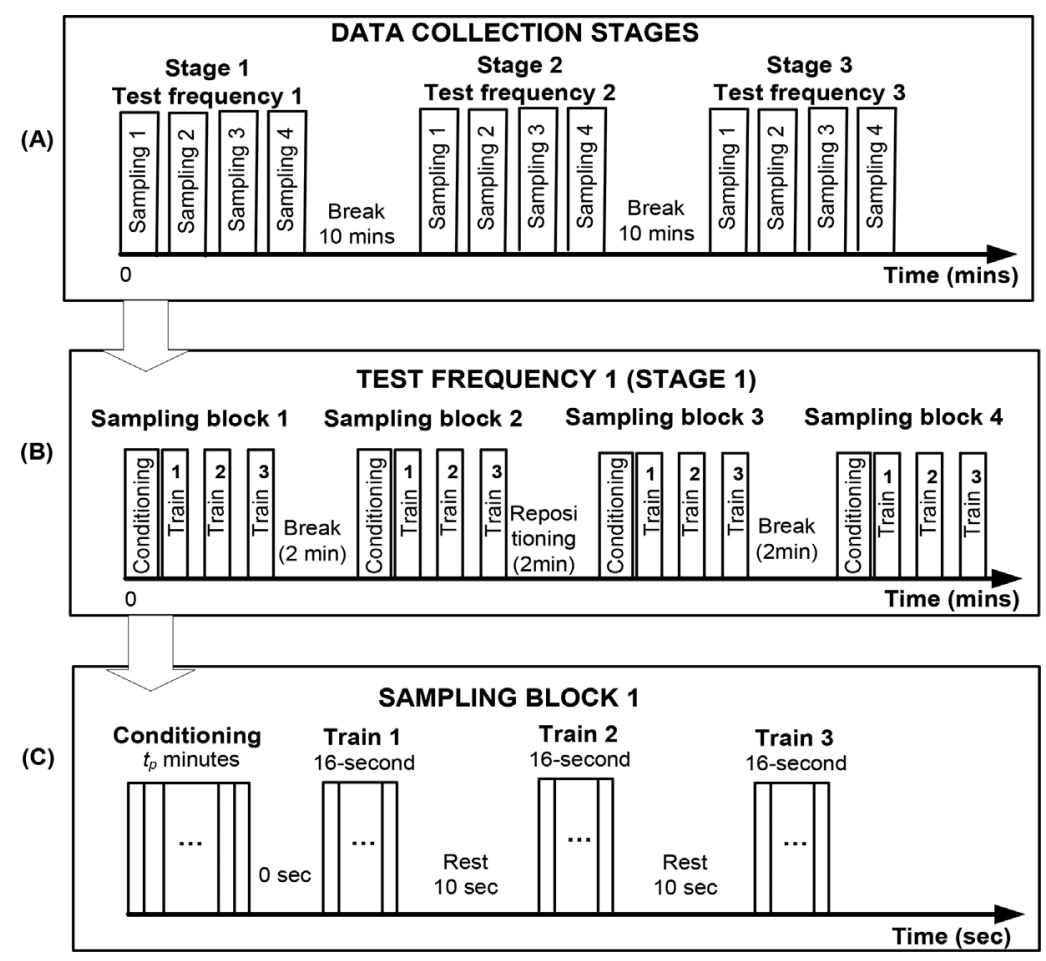

Figure 2 Schematic indication of stimulation procedures. (A) Three stimulation frequencies in separate test stages. (B) Muscle stimulation protocol during one data collection stage. Conditioning: continuous stimulation at a given stimulation frequency to reach a plateau in twitch force. Train: stimulation at each stimulation frequency with rest in between. (C) Stimulation during one sampling block. In addition, participants were repositioned between $2^{\text {nd }}$ and $3^{\text {rd }}$ sampling blocks.

electrodes would be placed was first shaved, and then skin was cleaned with alcohol and dried. On the basis of earlier recommendations on initial electrodes locations and probing procedures (Baker, McNeal, Benton, Bowman, \& Waters, 1993; Johnson et al., 1995a; Vanoncini, Holderbaum, \& Andrews, 2006), subsequent preparatory procedures were completed to identify the most effective stimulation site for each individual. Initially, negative electrodes were placed at the level of the iliac crest, and positive electrodes were placed at the level of the inferior margin of the rib cage (see Figure 1B). Stimulus intensity was then determined by increasing the stimulation current from $10 \mathrm{~mA}$ at each stimulation frequency (see below), until discomfort was reported or up to a max of $40 \mathrm{~mA}$, and it was subsequently reduced by $15 \%$. Discomfort/pain is related to the stimulation level, which is controlled by the pulse amplitude (current) and pulse duration. Measured stimulation responses are often a nonlinear function of either pulse duration or stimulation amplitude (Crago, Peckham, \& Thrope, 1980). During stimulation, it is important to minimize charge trans- fer to prevent damage to muscle tissue. At a given force level, a high stimulation amplitude requires less charge transfer per stimulation pulse, and charge transfer increases with increasing stimulation duration (Crago, Peckham, Mortimer, \& Van Der Meulen, 1974; Crago et al., 1980). As a compromise, we used a pulse duration of $0.3 \mathrm{~ms}$. Current-controlled stimulation (at $150 \mathrm{~V}$ ) was also chosen, as it was easier to control and may induce less discomfort compared to voltage-controlled stimulation (Merletti, Knaflitz, \& DeLuca, 1992). After identifying the stimulus intensity, the test muscles were "warmed up" using a 4-min stimulation block at the stimulation frequency. Finally, effective electrode sites were then determined by stimulating the muscle at each stimulation frequency and probing (i.e., adjusting the positive electrode locations) along superior-inferior direction until maximal evoked stimulation responses were measured using the load cell.

Each participant then completed three data collection stages (Figure 2A), where each stage involved one of the three stimulation frequencies $(2,5$, and $8 \mathrm{~Hz}$ ). A different presentation order was predefined 
for each participant using a Latin square, and a 10-min break was given after each stage. The same stimulation frequency and parameters were applied in the conditioning trains and in the subsequent measurement trains. Within each stage, stimulation responses were measured in four sampling blocks (Figure 2B) with a 2-min rest in between. Within each sampling block, a conditioning train was applied (Figure 2C) to first potentiate the muscles into a steady state (Desmedt \& Hainaut, 1968; Edwards et al., 1977; Kufel et al., 2002; Rassier \& MacIntosh, 2000). Each conditioning train was terminated when stimulation responses plateaued, determined in real-time by using a 1-s moving window mean of peak responses measured. This time-to-potentiation $\left(t_{\text {pot }}\right)$ was recorded along with the potentiated stimulation responses $\left(F_{\text {pot }}\right)$. Immediately after the conditioning train, muscle stimulation responses were measured during three 16-s stimulation trains (Figure 2C). Short breaks were provided between each train. To minimize potential effects of repositioning, however, participants were not allowed to leave the experimental fixture. After the second sampling block, participants were removed and repositioned in the experimental fixture. This was done to determine whether there were significant differences in stimulation responses.

\subsection{Data Processing and Analysis}

Stimulation responses were band-pass filtered (1.5$10 \mathrm{~Hz}$ ) to remove environmental and breathing artifacts (Beers, 2003). Peak responses from each stimulation pulse, within each train, were determined using MATLAB software (MathWorks, 2010a). Mean stimulation responses were the dependent measure in the repeated measures analyses of variance (RANOVAs). These were obtained as the means, across all peak stimulation responses recorded during the 16-s block of each train, excluding the three largest and three smallest values. Such a truncated (trimmed) mean was used as an estimate of central tendency, as it is less sensitive to outliers (Rothenberg, Fisher, \& Tilanus, 1964; Wilcox \& Keselman, 2003).

RANOVAs were used to identify any significant differences in mean stimulation responses with respect to stimulation frequency, sampling block, and stimulation train. Where relevant, significant effects were further investigated using Tukey's honestly significant difference post hoc analyses. RANOVA was also used to identify any significant differences in $t_{\text {pot }}$ related to stimulation frequency and sampling block. In all analyses, a $p$ value of $<0.05$ was considered statistically significant.

The reliability of stimulation responses was evaluated both within and between sampling blocks for each of the three stimulation frequencies. To allow for comparisons of stimulation responses within and between participants, stimulation responses measured within each conditioning train and twitch train were normalized to stimulation responses collected after muscle conditioning within the first sampling block. This approach was used, since $F_{\text {pot }}$ was expected to be the most stable response measured. Reliability was evaluated using intraclass correlation coefficients (ICC) and was determined separately for each of the three stimulation frequencies. Within sampling blocks reliability was determined by calculating ICC using data obtained from the three stimulation trains. Reliability between sampling blocks was evaluated by calculating ICC from mean stimulation responses in each train and across all four sampling blocks. ICC was also calculated using mean stimulation responses across all three trains to evaluate reliability between sampling blocks. Finally, ICC was used to assess the reliability of stimulation responses before and after repositioning the participant in the experimental fixture. ICCs were qualitatively interpreted [Cicchetti and Sparrow, 1981, as poor (0.00-0.39), fair (0.40-0.59), good (0.60-0.74), or excellent (0.75-1.00)].

\section{RESULTS}

Stimulation responses were successfully evoked using all three stimulation frequencies (Figure 3). In general, trunk movements and stimulation responses in response to stimulation at $2 \mathrm{~Hz}$ were more noticeable visually, compared to the other two frequencies. Stimulation at $2 \mathrm{~Hz}$ resulted in the largest stimulation responses, mean $(S D)=30.8(8.8) \mathrm{N}$, whereas stimulation at $5 \mathrm{~Hz}$, mean $(S D)=19.6(3.3) \mathrm{N}$, and $8 \mathrm{~Hz}$, mean $(S D)=19.7(4.5) \mathrm{N}$, yielded comparable but smaller responses. There was a significant effect of stimulation frequency, $F(2,175)=134.9, p<0.01$, on stimulation responses (Figure 4). No significant main effects of stimulation train, $F(2,175)=0.4, p=0.65$, or sampling block, $F(3,175)=0.9, p=0.34$, were evident, or any interaction effects of stimulation frequency, stimulation train, or sampling block, $p>0.91$.

Stimulation frequency also significantly, $F(2,60)=$ 17.3, $p<0.01$, affected $t_{\text {pot }}$, which was longest with 


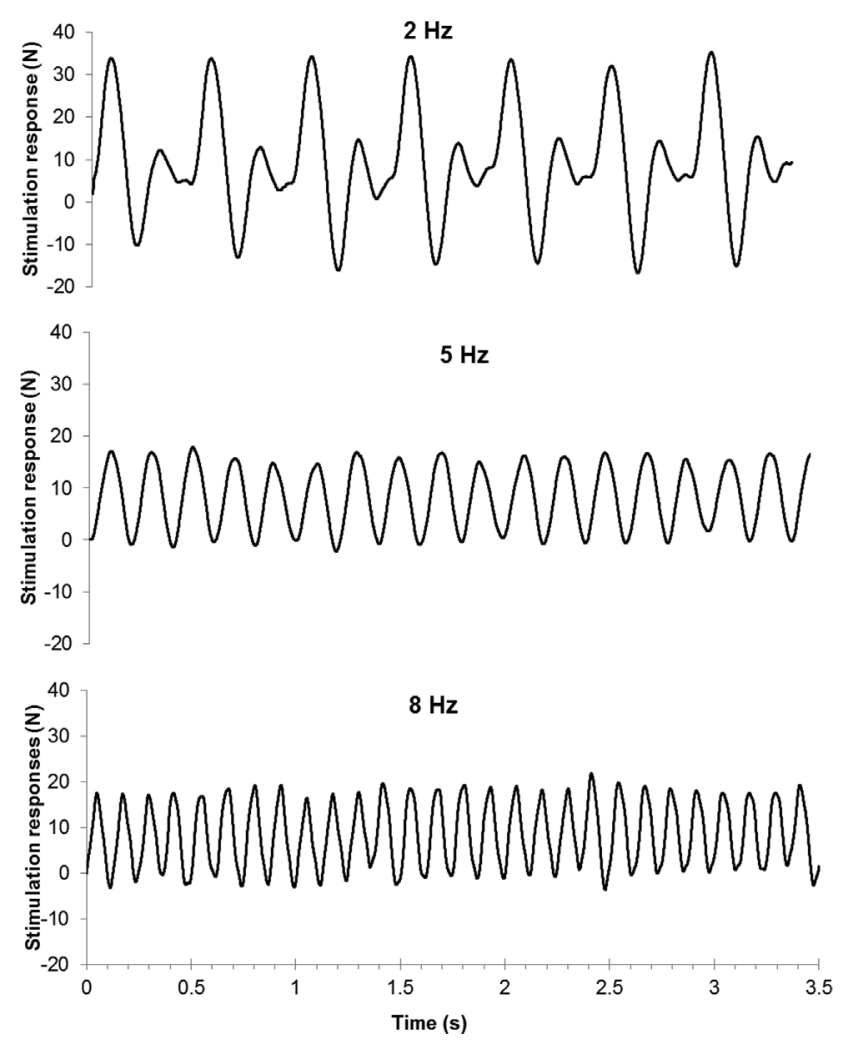

Figure 3 Sample stimulation responses resulting from three different stimulation frequencies. Positive $(+)$ values represent forces recorded from the load cell during trunk extension caused by muscle stimulation (i.e., positive values indicate load cell tension). Potential sources of the smaller, secondary negative and positive peaks, in response to $2 \mathrm{~Hz}$ stimulation, are discussed in the text.

$2 \mathrm{~Hz}$ stimulation $($ mean $=261 \mathrm{~s})$, compared to 5 or $8 \mathrm{~Hz}($ mean $=85 \mathrm{~s})$. Differences in $t_{\text {pot }}$ between the four sampling blocks were not significant, $F(3,60)=1.2$, $p=0.32$ ), and there was not a significant effect of the Frequency $\times$ Sampling Block interaction, $F(6,60)=$ $0.9, p=0.45$. Reliability of stimulation responses within each sampling block was in the excellent range $(>0.85)$ for all combinations of stimulation frequency
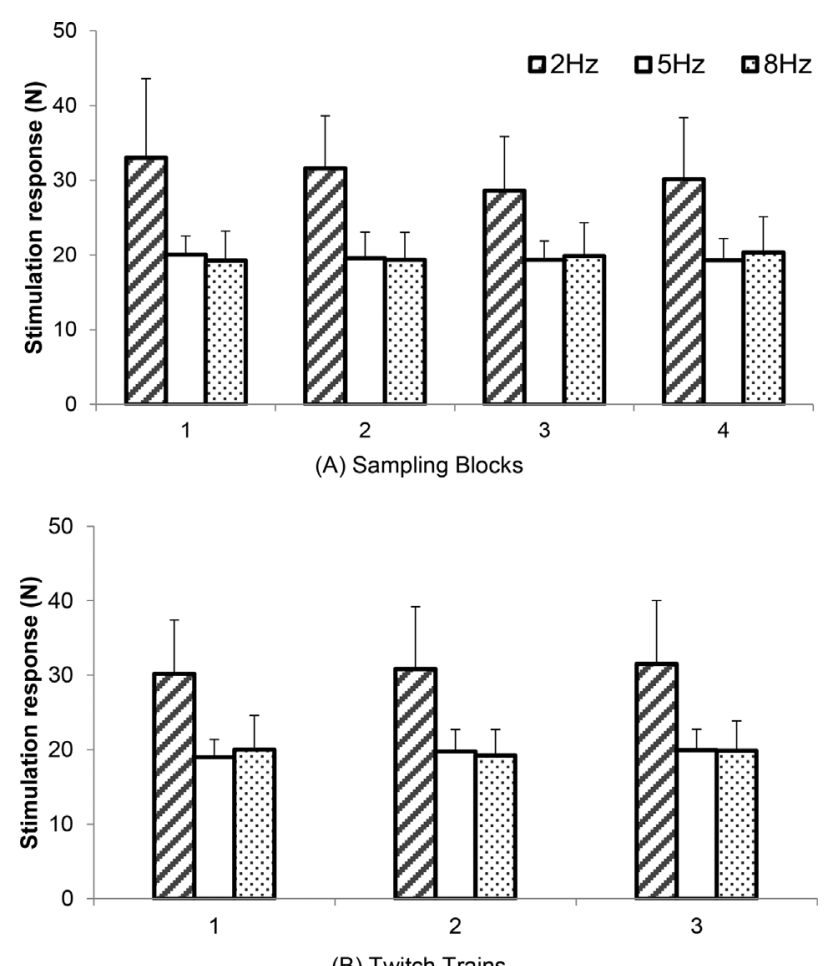

Figure 4 Mean stimulation responses (error bars indicated SDs) to the three stimulation frequencies during four sampling blocks (A) and three trains (B). Stimulation responses (forces) measured at $2 \mathrm{~Hz}$ were significantly $(p<0.05)$ larger than the other two stimulation frequencies in all cases.

and sampling block (Table 1). Qualitatively, reliability within sampling blocks was highest for stimulation at $2 \mathrm{~Hz}$ and consistent across the four sampling blocks. Excellent reliability was also evident between sampling blocks, with the highest levels found for stimulation at $8 \mathrm{~Hz}$ and the lowest at $2 \mathrm{~Hz}$. This same pattern was evident for the reliability of mean stimulation responses between sampling blocks. Repositioning participants did not substantially influence reliability (Table 2). Specifically, ICCs in each of the three trains were excellent, with one exception $(2 \mathrm{~Hz}$, Train 3$)$.

TABLE 1. Intraclass Correlation Coefficients (ICC) Values for Three Stimulation Frequencies

\begin{tabular}{|c|c|c|c|c|c|c|c|c|}
\hline & \multicolumn{4}{|c|}{ Within-SP ICC } & \multicolumn{3}{|c|}{ Between-SP ICC } & \multirow[b]{2}{*}{ Between-SP Mear } \\
\hline & SP 1 & SP 2 & SP 3 & SP 4 & Train 1 & Train 2 & Train 3 & \\
\hline $2 \mathrm{~Hz}$ & 0.96 & 0.93 & 0.91 & 0.96 & 0.84 & 0.83 & 0.81 & 0.84 \\
\hline $5 \mathrm{~Hz}$ & 0.89 & 0.94 & 0.85 & 0.89 & 0.88 & 0.91 & 0.93 & 0.91 \\
\hline $8 \mathrm{~Hz}$ & 0.91 & 0.89 & 0.94 & 0.84 & 0.96 & 0.95 & 0.89 & 0.96 \\
\hline
\end{tabular}

Notes: Determined within sampling blocks (Within-SP, for four sampling blocks), between sampling blocks (Between-SP, for three trains), and for mean twitch forces between four sampling blocks (Between-SP Mean). 
TABLE 2. ICC Values before and after Repositioning a Participant in the Experimental Fixture

\begin{tabular}{lccc}
\hline & \multicolumn{3}{c}{ Repositioning ICC } \\
\cline { 2 - 4 } & Train 1 & Train 2 & Train 3 \\
\hline $2 \mathrm{~Hz}$ & 0.87 & 0.84 & 0.76 \\
$5 \mathrm{~Hz}$ & 0.94 & 0.82 & 0.94 \\
$8 \mathrm{~Hz}$ & 0.97 & 0.88 & 0.97 \\
\hline
\end{tabular}

These values were generally comparable with overall reliability between sampling blocks (Table 1), both in terms of magnitude and the influence of stimulation frequency.

\section{DISCUSSION}

The objective of this study was to develop a method to measure stimulation responses of the lumbar trunk extensors in the context of seated postures. Muscle stimulation responses were successfully generated across all participants in response to all three stimulation frequencies. Stimulation responses collected at $2 \mathrm{~Hz}$ in particular resulted in a mean response of $32 \mathrm{~N}$, comparable to a value of $35 \mathrm{~N}$ in an earlier study (Vanoncini et al., 2006). Although the test condition was different than what was used here, the results from Vanoncini et al. (2006) are considered to at least provide relevant information regarding the magnitude of stimulation responses in a seated posture. All three frequencies of stimulation produced measurable responses to muscle contractions, and no substantial pain or skin irritation was either noticed or reported. Rather, participants indicated only minor discomfort caused by the stimulation, and some minor skin reddening was evident under the stimulation electrodes, which is considered a normal reaction to the long stimulation sessions involved (Baker et al., 1993).

As an important aspect of stimulation, muscle potentiation, was addressed. Potentiation is considered to be a result of phosphorylation of the myosin light chains and depends on the fiber type (Hanson, 1974; Houston, Green, \& Stull, 1985). Specifically, Type II fibers are selectively and preferentially activated before Type I fibers by stimulation (Hanson, 1974; Sinacore et al., 1990). The lumbar extensors (e.g., the erector spinae and multifidus) have a high proportion of Type I fibers. However, the percentage of Type II fibers can reach 35-50\% (Mannion et al., 1997; Thorstensson \& Carlson, 1987), which emphasizes the importance of potentiating the muscle first while using stimulation to measure stimulation responses from these muscles. In the current study, twitch potentiation was observed in all participants within the first 85-261 s of the conditioning trails. Compared to the $2 \mathrm{~Hz}$ stimulation frequency, less time was required to reach a steady state with 5 and $8 \mathrm{~Hz}$ frequencies. This inverse relationship between time-to-potentiation and stimulation frequency is consistent with existing studies of the knee extensors (Binder-Macleod, Dean, \& Ding, 2002; Eom, Watanabe, Hoshimiya, \& Khang, 2002) and ulnar nerve (Griffin \& Mettler, 2010). Eom et al. (2002) also indicated in their studies that the degree of force enhancement during potentiation decreased with the stimulation frequency, which was also observed in the current study. During muscle conditioning, substantial enhancements of stimulation responses were observed using all three test frequencies; approximate increases were 50,30 , and $30 \%$ in response to 2,5 , and $8 \mathrm{~Hz}$ stimulation, respectively. This again indicates the need for potentiation since measurements of stimulation responses without potentiation may introduce large variability to measured stimulation responses (Eom et al., 2002; Johnson et al., 1995a). As found here, muscle stimulation can lead to variable (increasing) magnitudes of stimulation responses $(30 \sim 50 \%)$ before reaching a steady state. No consistent pattern of potentiation in stimulated responses was found in the second or third trains within a sampling block. In a few cases, though, a fast (within first two to three stimulation pulses) and slight increase $(\leq 5 \%)$ in stimulation responses were observed at the start of these trains. Any effects of such increases were minimized, however, during calculation of mean stimulation responses using truncated means. Since mean stimulation responses measured between several sampling blocks (Figure 4) were consistent, the conditioning train appeared successful at achieving a steady state in terms of stimulation responses, and it likely did not introduce any muscle fatigue.

The reliability of evoked stimulation responses was assessed in several ways. In general, all three stimulation frequencies exhibited excellent reliability in terms of stimulation responses within and between sampling blocks. Among the three frequencies, within-sampling block reliability for $2 \mathrm{~Hz}$ was somewhat higher than the other two, indicating that potentiated twitches at $2 \mathrm{~Hz}$ were more stable in the three stimulation trains. However, in comparison to 5 and $8 \mathrm{~Hz}$ stimulation, relatively larger trunk movements were observed in response to 
the $2 \mathrm{~Hz}$ stimulation. Since the seated posture was not precisely controlled between different sampling blocks, the sitting position at each sampling block may have varied. Different sitting positions may result in different trunk movement pattern and such differences may have contributed to the somewhat lower reliability between different sampling blocks. Since $2 \mathrm{~Hz}$ stimulation provide more stable and larger stimulation responses, it is reasonable to expect more successful measurements in practical applications (e.g., to assess muscle fatigue). Stimulation at 5 and $8 \mathrm{~Hz}$, in contrast, though it resulted in higher ICC values between sampling blocks, also yielded stimulation responses that were relatively lower in magnitude and less stable over the three stimulation trains.

Four potential difficulties need to be acknowledged. First, the most effective location of the stimulation electrode was achieved using protocols derived from earlier work (Baker et al., 1993; Vanoncini et al., 2006). However, isolation of different muscle groups may not be practically achievable. The lumbar extensors can be functionally divided into several different muscle groups, such as the erector spinae and multifidus, with the muscles structured in different layers making it difficult to identify that the exact target muscle group has been stimulated in vivo. However, stimulation response measurements here were done with participants sitting in the experimental fixture in a relatively controlled posture, which should ensure that the same muscles were stimulated at a similar electrode location within each sampling block. Therefore, the potential effects of changes in muscle "cross-talk" and in muscle geometry were assumed to be minimal. In addition, unlike voluntary muscle contraction, muscle contraction induced by stimulation does not follow the size principle, and muscle fibers are instead recruited without obvious sequencing related to fiber types (Gregory \& Bickel, 2005; Kubiak, Whitman, \& Johnston, 1987). Instead, both slow and fast muscle fibers are nonselectively activated during muscle stimulation regardless of force levels (Gregory \& Bickel, 2005). As such, the potential effect of differing recruitment patterns can be minimized through stimulation. Therefore, for future applications, it is reasonable to assume that any measured changes in stimulation responses using these procedures are (predominantly) the result of changes in muscle contractile status (e.g., muscle fatigue) versus changes in muscle geometry and/or recruitment pattern. Further work will be completed to quantify the potential effects of trunk flexor and extensor activ- ities on measured stimulation responses. In addition, it remains to determine whether stimulation responses remain reliable over longer blocks of measurement. For example, there may be important influences of diurnal, temperature, or electrode changes.

Second, the seated posture was not precisely controlled between sampling blocks, and the exact postures may have varied between different sampling blocks. Large postural changes could have introduced variability in measured stimulation responses. One reason to select a sitting position for muscle stimulation is to match the conditions (e.g., posture) during the actual tasks we plan in future studies (of seated work tasks). By using similar conditions, physiological properties of muscles (e.g., muscle length) and levels of voluntary contraction can be kept relatively consistent and thereby help in distinguishing fatigue from potential confounding influences. Therefore, the effect of sitting postures could perhaps be minimized by controlling participant postures more precisely within and between different sampling blocks. In addition, monitoring trunk angles (e.g., using a motion tracking system) may facilitate methods to account for changes in measured stimulation responses caused by postural differences.

Third, voluntary contraction levels of the trunk flexors and extensors, as postural muscles, were controlled but not eliminated during muscle stimulation. Thus, the potential coexistence of muscle stimulation and voluntary contraction may have had a net effect on measured stimulation responses. Instead of pure twitch forces, the current methods provide a measure of net responses from both muscle stimulation and voluntary contraction of trunk flexors and extensors. Trunk extensors were monitored (real-time EMG) throughout the current study, to minimize the levels of voluntary contraction. Qualitatively, this monitoring indicated relatively low and consistent levels of contraction during the sampling blocks. In addition, the sitting posture adopted during the sampling blocks placed the lumbar spine in flexion; this placed the lumbar motion segments beyond a neutral posture and activation of the trunk extensors was thus predominant. Thus, while potential effects of voluntary contraction were not eliminated, these were considered relatively small in the current study. Further work will be completed to identify the effects of trunk posture on measured stimulation responses, and these results can be used to account for the effects of postural changes on measured stimulation responses. 
Fourth, the potential contribution of voluntary muscle contraction to stimulation responses should not be neglected. Additional voluntary muscle contractions, above that needed to maintain posture, were not observed during pilot work. However, voluntary contractions likely contributed to the negative and secondary positive peaks found in response to $2 \mathrm{~Hz}$ stimulation (see Figure 3) and may have also contributed to the initial positive peaks (that were the focus of the current analysis). More generally, these secondary peaks may also have resulted from contributions of reflexive behaviors or from the contribution of trunk momentum (e.g., a "rebounding" effect following trunk displacement induced by stimulation). Future work is needed to better isolate the specific responses to stimulation, which would provide improved estimates of muscle status (e.g., fatigue).

Our long-term goal is to use muscle stimulation for detecting lumbar extensors fatigue during prolonged sitting. The developed method will be used to measure muscle fatigue in a future study, specifically to assess situations involving prolonged sitting. At present, however, this approach may be limited to use in a laboratory environment, given the needs for a special seat and instrumentation. The current results suggest that stimulation at $2 \mathrm{~Hz}$ can provide suitable stimulation responses for this purpose. The higher responses captured using $2 \mathrm{~Hz}$ are considered beneficial, to allow for better separation of the muscle responses to stimulation from any background noise. Further, stimulation at this frequency required reasonable time blocks for potentiation $(\sim 4 \mathrm{~min})$ and evoked stimulation responses had excellent reliability. The current results indicated high levels of reliability within and between sampling blocks and trains for the protocol. While this suggests that only a single sampling train may be required, multiple sampling trains are recommended in future work to enhance the stability of obtained measures.

\section{References}

Baker, L., McNeal, D., Benton, L., Bowman, B., \& Waters, R. (1993). Neuromuscular electrical stimulation: A practical guide (3rd ed.). Downey, CA: Rancho Rehabilitation Engineering Program, Rancho Los Amigos Medical Center.

Beers, M. H. (2003). The Merck manual of medical information (2nd Home ed.). Whitehouse Station, NJ: Merck Research Laboratories.
Binder-Macleod, S. A., Dean, J. C., \& Ding, J. (2002). Electrical stimulation factors in potentiation of human quadriceps femoris. Muscle and Nerve, 25(2), 271-279. Binder-Macleod, S. A., \& Snyder-Mackler, L. (1993). Muscle fatigue: Clinical implications for fatigue assessment and neuromuscular electrical stimulation. Physical Therapy, 73(12), 902-910.

Blangsted, A. K., Sjøaard, G., Madeleine, P., Olsen, H. B., \& Søaard, K. (2005). Voluntary low-force contraction elicits prolonged low-frequency fatigue and changes in surface electromyography and mechanomyography. Journal of Electromyography and Kinesiology, 15(2), 138-148.

Byström, S., \& Kilbom, Å. (1991). Electrical stimulation of human forearm extensor muscles as an indicator of handgrip fatigue and recovery. European Journal of Applied Physiology and Occupational Physiology, 62(5), 363-368.

Cicchetti, D. V., \& Sparrow, S. A. (1981). Developing criteria for establishing interrater reliability of specific items: Applications to assessment of adaptive behavior. American Journal of Mental Deficiency, 86(2), 127-137. Cooper, R. G., Edwards, R. H., Gibson, H., \& Stokes, M. J. (1988). Human muscle fatigue: Frequency dependence of excitation and force generation. Journal of Physiology, 397, 585-599.

Crago, P., Peckham, P., Mortimer, J., \& Van Der Meulen, J. (1974). The choice of pulse duration for chronic electrical stimulation via surface, nerve, and intramuscular electrodes. Annals of Biomedical Engineering, 2(3), 252-264.

Crago, P. E., Peckham, P. H., \& Thrope, G. B. (1980). Modulation of muscle force by recruitment during intramuscular stimulation. IEEE Transactions on Biomedical Engineering, BME-27(12), 679-684.

Cram, J. R., \& Rommen, D. (1989). Effects of skin preparation on data collected using an EMG muscle-scanning procedure. Applied Psychophysiology and Biofeedback, 14(1), 75-82.

Dankaerts, W. P., O'Sullivan, P. P., Burnett, A. P., \& Straker, L. P. (2006). Differences in sitting postures are associated with nonspecific chronic low back pain disorders when patients are subclassified. Spine, 31(6), 698-704.

Davies, C. T., \& White, M. J. (1982). Muscle weakness following dynamic exercise in humans. Journal of Applied Physiology, 53(1), 236-241.

Desmedt, J. E., \& Hainaut, K. (1968). Kinetics of myofilament activation in potentiated contraction: Staircase phenomenon in human skeletal muscle. Nature, 217(5128), 529-532.

Edwards, R. H., Hill, D. K., Jones, D. A., \& Merton, P. A. (1977). Fatigue of long duration in human skeletal muscle after exercise. Journal of Physiology, 272(3), 769-778. 
Eom, G., Watanabe, T., Hoshimiya, N., \& Khang, G. (2002). Gradual potentiation of isometric muscle force during constant electrical stimulation. Medical and Biological Engineering and Computing, 40(1), 137-143.

Fitch, S., \& McComas, A. (1985). Influence of human muscle length on fatigue. Journal of Physiology, 362, 205-213.

Ford, E. S., Kohl, H. W., Mokdad, A. H., \& Ajani, U. A. (2005). Sedentary behavior, physical activity, and the metabolic syndrome among U.S. adults. Obesity, 13(3), 608-614.

Fowles, J. R., \& Green, H. J. (2003). Coexistence of potentiation and low-frequency fatigue during voluntary exercise in human skeletal muscle. Canadian Journal of Physiology and Pharmacology, 81(12), 1092-1100.

Gamet, D., Duchene, J., Garapon-Bar, C., \& Goubel, F. (1993). Surface electromyogram power spectrum in human quadriceps muscle during incremental exercise. Journal of Applied Physiology, 74(6), 2704-2710.

Gregory, C. M., \& Bickel, C. S. (2005). Recruitment patterns in human skeletal muscle during electrical stimulation. Physical Therapy, 85(4), 358-364.

Griffin, L., \& Mettler, J. A. (2010). What are the stimulation parameters that affect the extent of twitch force potentiation in the adductor pollicis muscle? European Journal of Applied Physiology, 110(6), 1235-1242.

Hakala, P. T., Rimpela, A. H., Saarni, L. A., \& Salminen, J. J. (2006). Frequent computer-related activities increase the risk of neck-shoulder and low back pain in adolescents. European Journal of Public Health, 16(5), 536-541.

Hallal, P. C., Andersen, L. B., Bull, F. C., Guthold, R., Haskell, W., \& Ekelund, U. (2012). Global physical activity levels: Surveillance progress, pitfalls, and prospects. Lancet, 380(9838), 247-257.

Hanson, J. (1974). The effects of repetitive stimulation on the action potential and the twitch of rat muscle. Acta Physiologica Scandinavica, 90(2), 387-400.

Houston, M. E., Green, H. J., \& Stull, J. T. (1985). Myosin light chain phosphorylation and isometric twitch potentiation in intact human muscle. Pflügers Archiv European Journal of Physiology, 403(4), 348-352.

Johnson, P. W. (1998). The development, characterization and implementation of a technique to measure muscle fatigue during computer use. University of California, Berkeley.

Johnson, P. W., Lehman, S. L., \& Rempel, D. M. (1995a). Measuring low frequency fatigue with 2 Hertz stimulation. I. Stimulus-related potentiation effects. Paper presented at the Engineering in Medicine and Biology Society, IEEE 17th Annual Conference.

Johnson, P. W., Lehman, S. L., \& Rempel, D. M. (1995b). Measuring low frequency fatigue with 2 Hertz stimulation. II. Muscle fatigue results. Paper presented at the Engineering in Medicine and Biology Society, IEEE 17th Annual Conference.

Johnson, P. W., Lehman, S. L., \& Rempel, D. M. (1996). Measuring muscle fatigue during computer mouse use. Paper presented at the Engineering in Medicine and Biology Society. Bridging Disciplines for Biomedicine. Proceedings of the 18th Annual International Conference of the IEEE.

Kolich, M., Mohamed, A. I., \& Taboun, S. M. (2001). Electromyographic comparison of two lumbar support mechanisms intended for automotive seating applications. Proceedings of the Institution of Mechanical Engineers. Part D, Journal of Automobile Engineering, 215(7).

Kubiak, R. J., Whitman, K. M., \& Johnston, R. M. (1987). Changes in quadriceps femoris muscle strength using isometric exercise versus electrical stimulation. Journal of Orthopaedic and Sports Physical Therapy, 8(11), 537-541.

Kufel, T. J., Pineda, L. A., \& Mador, M. J. (2002). Comparison of potentiated and unpotentiated twitches as an index of muscle fatigue. Muscle and Nerve, 25(3), 438-444.

Mannion, A. F., Dumas, G. A., Cooper, R. G., Espinosa, F. J., Faris, M. W., \& Stevenson, J. M. (1997). Muscle fibre size and type distribution in thoracic and lumbar regions of erector spinae in healthy subjects without low back pain: Normal values and sex differences. Journal of Anatomy, 190(4), 505-513.

McLean, L., \& Goudy, N. (2004). Neuromuscular response to sustained low-level muscle activation: Within- and between-synergist substitution in the triceps surae muscles. European Journal of Applied Physiology, 91(2), 204-216.

Merletti, R., Knaflitz, M., \& DeLuca, C. J. (1992). Electrically evoked myoelectric signals. Critical Reviews in Biomedical Engineering, 19(4), 293-340.

Mork, P. J., \& Westgaard, R. H. (2005). Long-term electromyographic activity in upper trapezius and low back muscles of women with moderate physical activity. Journal of Applied Physiology, 99(2), 570-578.

Mork, P. J., \& Westgaard, R. H. (2009). Back posture and low back muscle activity in female computer workers: A field study. Clinical Biomechanics 24(2), 169-175.

Nagata, S., Arsenault, A. B., Gagnon, D., Smyth, G., \& Mathieu, P. A. (1990). EMG power spectrum as a measure of muscular fatigue at different levels of contraction. Medical \& Biological Engineering \& Computing, 28(4), 374-378.

NIOSH. (1997). Musculoskeletal disorders and workplace factors: A critical review of epidemiologic evidence for work-related musculoskeletal disorders of the neck, upper extremity, and low back. DHHS Pub. No. 97141 . 
Oberg, T., Sandsjo, L., \& Kadefors, R. (1994). Subjective and objective evaluation of shoulder muscle fatigue. Ergonomics, 37(8), 1323-1333.

Oberg, T., Sandsjo, L., Kadefors, R., \& Larsson, S. E. (1992). Electromyographic changes in work-related myalgia of the trapezius muscle. European Journal of Applied Physiology and Occupational Physiology, 65(3), 251-257.

O'Sullivan, P. B., Mitchell, T., Bulich, P., Waller, R., \& Holte, J. (2006). The relationship beween posture and back muscle endurance in industrial workers with flexion-related low back pain. Manual Therapy, 11(4), 264-271.

Pleis, J. R., Lucas, J. W., \& Ward, B. W. (2009, December). Summary health statistics for U.S. adults: National Health Interview Survey, 2008. Vital and Health Statistics. Series 10, Data from the National Health Survey (242), 1-157.

Rassier, D. E., \& MacIntosh, B. R. (2000). Coexistence of potentiation and fatigue in skeletal muscle. Brazilian Journal of Medical and Biological Research, 33, 499508.

Rothenberg, T. J., Fisher, F. M., \& Tilanus, C. B. (1964). A note on estimation from a Cauchy sample. Journal of the American Statistical Association, 59(306), 460-463.

Sinacore, D. R., Delitto, A., King, D. S., \& Rose, S. J. (1990). Type II fiber Activation with electrical stimulation: A preliminary report. Physical Therpy, 70(7), 416-422.

Sjøgaard, G., Kiens, B., Jørgensen, K., \& Saltin, B. (1986). Intramuscular pressure, EMG and blood flow during low-level prolonged static contraction in man. Acta Physiologica Scandinavica, 128(3), 475-484.

Small, S. C., \& Stokes, M. J. (1992). Stimulation frequency and force potentiation in the human adductor pollicis muscle. European Journal of Applied Physiology and Occuputational Physiology 65(3), 229-233.

Søgaard, K., Blangsted, A. K., Jørgensen, L. V., Madeleine, P., \& Sjøgaard, G. (2003). Evidence of long term muscle fatigue following prolonged intermittent contractions based on mechano- and electromyograms. Journal of Electromyography and Kinesiology, 13(5), 441450.
Sood, D., Nussbaum, M. A., \& Hager, K. (2007). Fatigue during prolonged intermittent overhead work: Reliability of measures and effects of working height. Ergonomics, 50(4), 497-513.

Thorstensson, A., \& Carlson, H. (1987). Fibre types in human lumbar back muscles. Acta Physiologica Scandinavica, 131(2), 195-202.

U.S. Department of Health and Human Services. (1996). Physical activity and health: A report of the Surgeon General. Atlanta, GA: U.S. Department of Health and Human Services, Public Health Service, CDC, National Center for Chronic Disease Prevention and Health Promotion.

van Dieën, J. H., de Looze, M. P., \& Hermans, V. (2001). Effects of dynamic office chairs on trunk kinematics, trunk extensor EMG and spinal shrinkage. Ergonomics, 44(7), 739-750.

van Dieën, J. H., Westebring-van der Putten, E. P., Kingma, I., \& de Looze, M. P. (2009). Low-level activity of the trunk extensor muscles causes electromyographic manifestations of fatigue in absence of decreased oxygenation. Journal of Electromyography and Kinesiology, 19(3), 398.

Vanoncini, M., Holderbaum, W., \& Andrews, B. J. (2006). Investigations on the biomechanics of the human trunk. Proceedings of the 24th IASTED International Conference on Biomedical Engineering. Anaheim, CA: ACTA Press.

Varo, J. J., Martinez-Gonzalez, M. A., de Irala-Estevez, J., Kearney, J., Gibney, M., \& Martinez, J. A. (2003). Distribution and determinants of sedentary lifestyles in the European Union. International Journal of Epidemiololgy, 32(1), 138-146.

Vollestad, N. K. (1997). Measurement of human muscle fatigue. Journal of Neuroscience Methods, 74(2), 219227.

Wilcox, R. R., \& Keselman, H. J. (2003). Modern robust data analysis methods: Measures of central tendency. Psychological Methods 8(3), 254-274.

Womersley, L., \& May, S. (2006). Sitting posture of subjects with postural backache. Journal of Manipulative and Physiological Therapeutics, 29(3), 213-218. 